\title{
Rat1p maintains RNA polymerase II CTD phosphorylation balance
}

\author{
SILVIA JIMENO-GONZÁLEZ, ${ }^{1}$ MANFRED SCHMID, FRANCISCO MALAGON, ${ }^{2}$ LINE LINDEGAARD \\ HAANING, ${ }^{3}$ and TORBEN HEICK JENSEN ${ }^{4}$ \\ Center for mRNP Biogenesis and Metabolism, Department of Molecular Biology and Genetics, Aarhus University, DK-8000, Aarhus, Denmark
}

\begin{abstract}
In S. cerevisiae, the $5^{\prime}-3^{\prime}$ exonuclease Rat1p partakes in transcription termination. Although Rat1p-mediated RNA degradation has been suggested to play a role for this activity, the exact mechanisms by which Rat1p helps release RNA polymerase II (RNAPII) from the DNA template are poorly understood. Here we describe a function of Rat1p in regulating phosphorylation levels of the C-terminal domain (CTD) of the largest RNAPII subunit, Rpb1p, during transcription elongation. The rat1-1 mutant exhibits highly elevated levels of CTD phosphorylation as well as RNAPII distribution and transcription termination defects. These phenotypes are all rescued by overexpression of the CTD phosphatase Fcp1p, suggesting a functional relationship between the absence of Rat1p activity, elevated CTD phosphorylation, and transcription defects. We also demonstrate that rat1-1 cells display increased RNAPII transcription kinetics, a feature that may contribute to the cellular phenotypes of the mutant. Consistently, the rat1-1 allele is synthetic lethal with the rpb1-E1103G mutation, causing increased RNAPII speed, and is suppressed by the rpb2-10 mutation, causing slowed transcription. Thus, Rat1p plays more complex roles in controlling transcription than previously thought.
\end{abstract}

Keywords: RNA polymerase II; CTD phosphorylation; transcription elongation; transcription termination

\section{INTRODUCTION}

The transcription cycle of RNAPII comprises three main phases-initiation, elongation, and termination, each of which is associated with specific phosphorylation patterns of the C-terminal domain (CTD) of Rpblp. The "CTD code" proposes that differential CTD phosphorylation regulates the recruitment of transcription and mRNA processing factors to the RNAPII machinery and is best understood for phosphorylation of serine2 (Ser2P) and serine5 (Ser5P) residues. During initiation, the CTD is hypophosphorylated, which allows binding of transcription initiation factors and assembly of the preinitiation complex (PIC). Promoter escape and early transcription elongation occur concomitantly with Ser5 phosphorylation, which facilitates pre-mRNA 5 -end capping and the stable transition from initiation to elongation (promoter release) (Rasmussen and Lis 1993; Komarnitsky et al. 2000; Schroeder et al. 2000; Kim et al. 2010). Ser5P is gradually removed during transcription elon-

\footnotetext{
${ }^{1}$ Present address: Departamento de Biología Molecular, Centro Andaluz de Biología Molecular y Medicina Regenerativa (CABIMER), E-41092, Seville, Spain

${ }^{2}$ Present address: Laboratory of Molecular Biology, Developmental Genetics Section, National Cancer Institute, Bethesda, MD 20892, USA

${ }^{3}$ Present address: Taconic Europe, DK-8600, Silkeborg, Denmark

${ }^{4}$ Corresponding author

E-mail thj@mb.au.dk

Article published online ahead of print. Article and publication date are at http://www.rnajournal.org/cgi/doi/10.1261/rna.041129.113.
}

gation, due to the actions of the CTD phosphatases Ssu72p and Rtr $1 \mathrm{p}$ and is only completely removed at the polyadenylation (pA) site (Kim et al. 2010; Bataille et al. 2012). Ser2P, which is absent during transcription initiation, gradually increases along transcription units and remains high until transcription termination. Ser2P plays a central role in coupling pre-mRNA processing to transcription elongation, and substitution of CTD-Ser2 residues to alanine impairs splicing and $3^{\prime}$ end processing in mammalian cells ( $\mathrm{Gu}$ et al. 2013). A major Ser2 kinase in Saccharomyces cerevisiae is Ctk1p, whose inactivation leads to strongly decreased Ser2P levels, a severely impaired recruitment of $3^{\prime}$ end processing factors and an accumulation of RNAPII at the $3^{\prime}$ ends of genes with weak pA sites (Ahn et al. 2004; Kim et al. 2010). Ser2P is removed during or after transcription termination by the CTDphosphatase Fcplp to prepare RNAPII for new initiation events (Kobor et al. 1999; Cho et al. 2001; Mandal et al. 2002). In addition, Fcp1p dampens Ser2P levels during normal transcription elongation as $f c p 1$ mutants display increased Ser2P levels inside genes (Archambault et al. 1997; Kobor et al. 1999; Cho et al. 2001; Ghosh et al. 2008).

C 2014 Jimeno-González et al. This article is distributed exclusively by the RNA Society for the first 12 months after the full-issue publication date (see http://rnajournal.cshlp.org/site/misc/terms.xhtml). After 12 months, it is available under a Creative Commons License (Attribution-NonCommercial 3.0 Unported), as described at http://creativecommons.org/licenses/by$\mathrm{nc} / 3.0 /$. 
Apart from coupling transcription with RNA processing, Ser2P may also influence the speed by which RNAPII proceeds through chromatin. For example, mammalian RNAPII, harboring Ser2 to alanine substitutions, shows decreased elongation rates (Gu et al. 2013). Furthermore, in human cells the speed of RNAPII increases toward the gene $3^{\prime}$ end, which could be related to increasing Ser2P (Danko et al. 2013). Similarly in S. cerevisiae, mutation of the multipurpose transcription factor Sublp leads to altered CTD phosphorylation levels and cotranscriptional recruitment of CTD kinases/phosphatases, which is paralleled by decreased transcription elongation rates (Calvo and Manley 2005; Garcia et al. 2010). Thus, although mechanistic details need to be elucidated, a link between CTD phosphorylation and transcription elongation appears to exist.

The mechanistic details underlying transcription termination are not well worked out. A current model postulates that Ratlp targets the $5^{\prime}$ end of the nascent downstream RNA fragment appearing after pA site cleavage. This causes, in an unknown fashion, the dissociation of RNAPII from the DNA template (Kuehner et al. 2011). RNAPII transcription stalling at or near the pA site may help in this process. CTD phosphorylation also impacts transcription termination. Most importantly, Ser2P facilitates binding of the pre-mRNA $3^{\prime}$ end processing-factor and transcription termination-factor Pcf11p and the Ratlp-cofactor Rtt103p (Lunde et al. 2011). The cotranscriptional recruitment of the Ser2P kinase Ctk1p ceases around the $\mathrm{pA}$ site, suggesting that Ser2P is lost in the downstream region (Mayer et al. 2010). However, to what extent Ser2P, or its removal, is functionally important for transcription termination in vivo is unclear. In addition to its role in transcription termination at gene $3^{\prime}$ ends, Ratlp also aids in terminating transcription events inside the gene body, provided that they are engaged with inefficiently capped pre-mRNA (Jimeno-Gonzalez et al. 2010).

The majority of the in vivo studies of Ratlp function rely on the thermosensitive rat1-1 mutant, the activity of which is poorly characterized. It contains a single mutation (Y657C) situated outside of the enzyme's exonuclease domain, and since the Rat1-1p protein is stable at the restrictive temperature, it may retain some exonuclease activity. Even so, growth and transcription termination phenotypes of rat $1-1$ cells are not rescued by the coexpression of the catalytically inactive rat1-D325A point mutant (Kim et al. 2004; Luo et al. 2006; Mayer et al. 2010). This has been taken as evidence that $5^{\prime}-3^{\prime}$ exonucleolysis is essential for Rat $1 p$ function and that this activity is, at least partly, compromised in the rat1-1 background.

Here, we find that overexpression of Fcplp suppresses the thermosensitivity of rat1-1. Surprisingly, rat1-1 cells not only exhibit a transcription termination defect but also display highly elevated CTD Ser2P levels as well as decreased RNAPII occupancy within genes. These phenotypes are all partially restored by Fcp1p overexpression. High Ser2P levels in rat $1-1$ cells are not due to decreased Fcplp levels around transcribed chromatin but rather to an increased cotranscriptional recruitment of Ctk1p. Finally, rat1-1 cells show increased transcription elongation rates. The data suggest that Ratlp plays a CTD-modulatory role during transcription elongation, which needs to be considered when interpreting molecular phenotypes of the rat1-1 mutant.

\section{RESULTS AND DISCUSSION}

\section{Increased phosphorylation of the Rpb1p CTD in rat1-1 cells}

To explore mechanisms used by Ratlp to promote transcription termination, we screened for multicopy suppressors of the rat1-1 mutant at its nonpermissive temperature of $34^{\circ} \mathrm{C}$ (see Materials and Methods). In addition to the RAT1 gene, we found that overexpression of the CTD Ser2P phosphatase, Fcplp, was able to rescue rat1-1 thermosensitivity at $34^{\circ} \mathrm{C}$ (Fig. 1A). This suggested that reduction of CTD Ser2P phosphorylation levels might help overcome growthlimiting defects of the rat1-1 mutant. We therefore analyzed the global CTD phosphorylation status of Rpb1p in the rat1-1 background with or without Fcp1p overexpression. Western blotting analysis of whole-cell extracts using antibodies recognizing CTD-Ser2P, -Ser5P, or -Ser7P residues demonstrated elevated Ser2P and Ser5P levels in the rat1-1 strain,
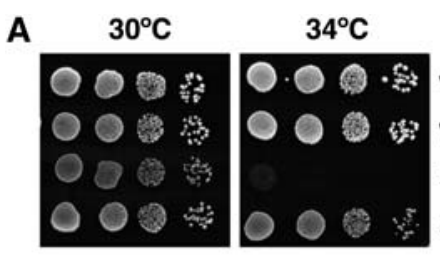

wt pRS425 wt pRS425-FCP1 rat1-1 pRS425 rat1-1 pRS425-FCP1

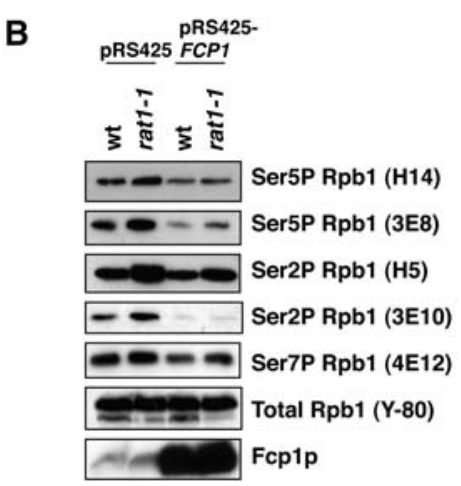

FIGURE 1. Excess Fcplp rescues growth deficiency of rat1-1 cells. $(A)$ Tenfold dilution series of wt and rat1-1 strains transformed with pRS425 or pRS425-FCP1 plasmids as indicated and spotted onto AA-Leu plates. Strains were grown for $3 \mathrm{~d}$ at $30^{\circ} \mathrm{C}$ or $34^{\circ} \mathrm{C}$. (B) Rpblp CTD phosphorylation status in whole-cell extracts measured by Western blotting analysis. Strains from $A$ were grown at $30^{\circ} \mathrm{C}$ in AA-Leu medium to an $\mathrm{OD}_{600}$ of 0.6 , followed by a subsequent incubation for $1 \mathrm{~h}$ at $34^{\circ} \mathrm{C}$, before being harvested for protein extraction. Membranes were probed with H14, H5, 3E8, 3E10, 4E12, Rpb1p (Y-80), or Fcp1p antibodies as indicated. 
whereas Ser7P levels were not affected (Fig. 1B). Both Ser2P and Ser5P levels were decreased by Fcplp overexpression.

\section{Fcp1p overexpression reduces elevated CTD-Ser2P levels at active chromatin in rat1-1 cells and partly suppresses related RNAPII distribution phenotypes}

We next tested whether increased CTD phosphorylation in rat1-1 cells was also manifested during active transcription and, if so, which impact excess Fcplp might have. For this analysis we utilized the Ser2P antibody $\mathrm{H} 5$, whose epitope is increased in rat1-1 cells and dampened to roughly wt levels upon Fcplp overexpression (Fig. 1B). Notably, H5 has highest affinity for a CTD Ser2P epitope containing neighboring Ser5P (Chapman et al. 2007). Chromatin immunoprecipitation (ChIP) experiments using $\mathrm{H} 5$ as well as Rpblp $(\mathrm{Y}-80)$ antibodies were carried out and immunoprecipitated chromatin was interrogated by PCR amplicons distributed at different positions along the reporter gene GAL1-YLR454W (Fig. 2A; Mason and Struhl 2005). In line with previous reports (Komarnitsky et al. 2000; Bataille et al. 2012), we found that in wt conditions, Ser2P levels were low at the promoter, increased in the coding region, and declined immediately after RNAPII passage of the pA site (Fig. 2B, left panel). In contrast, rat1-1 cells displayed abnormally high levels of Ser2P inside the YLR454W gene body, whereas the presence of this epitope disappeared downstream from the pA site. Such increased Ser2P levels were not simply due to the presence of more chromatin-associated RNAPII in rat1-1 cells as evidenced by ChIP assays conducted with the phosphorylation-insensitive Y-80 antibody (Fig. 2C, left panel). Indeed, the Ser2P/ Rpblp signal ratio increased robustly (up to sixfold) upon Ratlp mutation (Fig. 2D, left panel). A similar phenotype is observed at the PMA1 gene (Supplemental Fig. S1A,B), which, together with the Western blot analysis, suggests that increased CTD phosphorylation is a hallmark of transcription in rat1-1 cells. As predicted, Ser2P levels were reduced upon Fcplp overexpression, which restored high Ser2P ChIP signals of rat1-1 cells to close to wt levels (Fig. 2B,D, cf. right and left panels).
Which molecular phenotypes of rat1-1 cells may be associated with elevated CTD-Ser2P levels? Consistent with previous reports (Kim et al. 2004; West et al. 2004; Luo et al. 2006; Jimeno-Gonzalez et al. 2010), the rat1-1 mutation caused RNAPII transcription read-through of the YLR454 pA site (amplicons 8.5 and 9) (Fig. 2C, left panel, and E, right panel). Interestingly, RNAPII accumulated to similar levels near the pA site (8.2) in the wt and rat1-1 strains, despite lower levels within the YLR454W gene body of rat1-1 cells (2 to 8 regions) (Fig. 2C, left panel). A possible interpretation
A

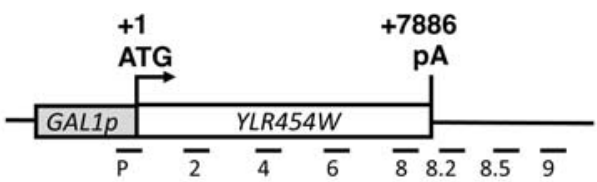

B

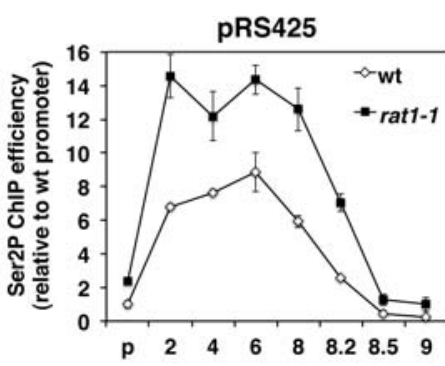

C

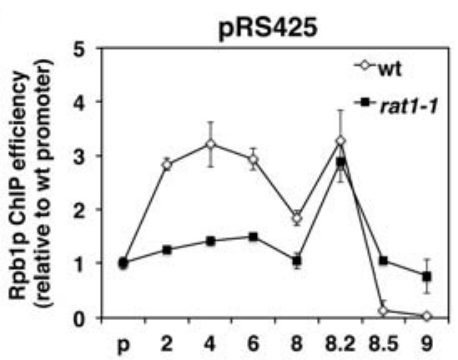

D

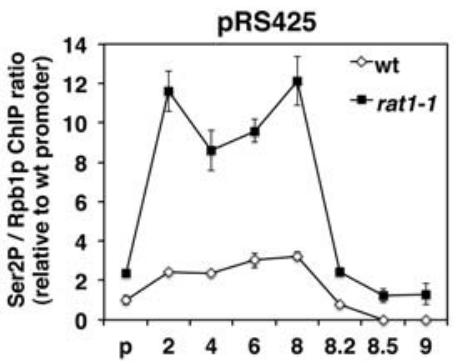

E
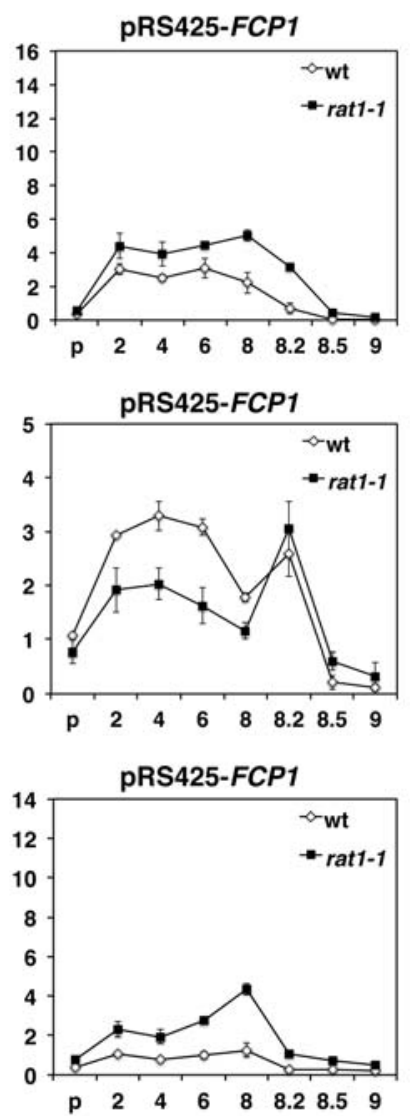
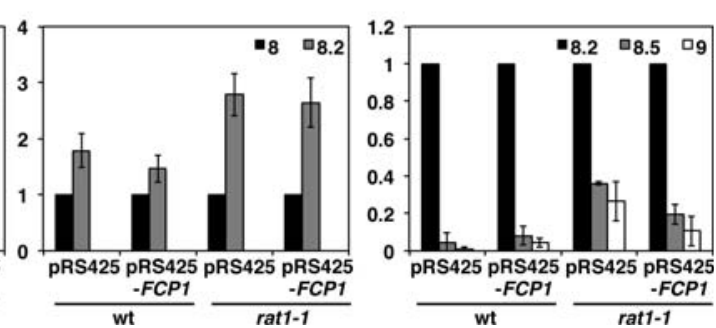

FIGURE 2. (Legend on next page) 
of these data is that RNAPII stalls in the vicinity of the pA site to be targeted by Ratlp-dependent "torpedo" termination (Kim et al. 2004; Luo et al. 2006). Failure to terminate efficiently in the rat $1-1$ context leads to an enhanced accumulation of stalled RNAPII versus transcribing polymerases within the gene body (Fig. 2E, middle and right panel). In addition, only in mutant cells can RNAPII escape the stalling site to "read-through" and reach downstream regions 8.5 and 9.

A strong rat1-1-dependent phenotype could also be observed at the YLR454W 5' end; while RNAPII immunoprecipitation (IP) efficiencies at the promoter were similar in the two backgrounds, only $\sim 40 \%-50 \%$ of RNAPII levels were detected along the YLR454W gene body in rat1-1 compared with wt cells (Fig. 2C, left panel, cf. amplicon p with 2, 4, 6, and 8; Fig. 2E, left panel). A similar phenotype was described for the PMA1 gene in previous reports (Kim et al. 2004; Kawauchi et al. 2008). This effect may be explained by the faster transcription elongation rates in the rat1-1 mutant (see below). Importantly, Fcplp overexpression partially suppressed both the decreased levels of RNAPII in the gene body (ratio between promoter and gene body ChIP amplicons) (Fig. 2E, left panel) and the transcription read-through (ChIP signals at amplicons 8.5 and 9) (Fig. 2E, right panel) phenotype of the rat1-1 mutant, without markedly changing levels of RNAPII "pA site stalling” (8.2) (Fig. 2E, middle panel).

To rule out that the observed effects would be specific for the GAL1-YLR454W reporter, we also analyzed RNAPII distribution at various positions along the PMA1 gene (Supplemental Fig. S1A). Gratifyingly, both decreased polymerase in the gene relative to the promoter (Supplemental Fig. S1C, cf. 5 to middle amplicons), and transcription termination (Supplemental Fig. S1C, cf. 3' UTR and T1 amplicons) phenotypes of rat1-1 cells were observed at the PMA1 locus. Note that RNAPII pA site-stalling and read-through effects are difficult to distinguish for the PMA1 gene due to the presence of multiple pA sites spread over a 450-bp region
(Ozsolak et al. 2010). Even so, a clear suppression of both gene $5^{\prime}$ - and $3^{\prime}$-end defects could be measured upon Fcplp overexpression (Supplemental Fig. S1B).

We previously reported that rat1-1 thermosensitivity is also suppressed by the rpb1-N488D allelic background conferring slow transcription rates (Jimeno-Gonzalez et al. 2010). The molecular mechanism underlying this suppression was not elucidated. Interestingly, introduction of the rpb1-N488D mutation caused decreased Ser2P levels over the YLR454W reporter gene in both the wt and rat1-1 mutant contexts (Supplemental Fig. S2A, cf. left and right panels). This effect was not due to a change in RNAPII levels in the rpb1-N488D background (Supplemental Fig. S2B). Since both suppressors of rat1-1 thermosensitivity, the rpbl$N 488 D$ allele and high-copy FCP1, are linked to decreased CTD phosphorylation, this may underlie the growth defect of rat $1-1$ cells. Indeed, the $r p b 1-N 488 D$ allele also suppressed the decreased RNAPII levels inside the gene and transcription read-through phenotypes of rat1-1 (Supplemental Fig. 2C, left and right panels; Jimeno-Gonzalez et al. 2010). Taken together, these data suggest that abnormally high Ser2P levels contribute to altered transcription elongation and termination in rat1-1 cells. Reducing Ser2P levels by excess Fcp1p or the rpb1-N488D mutant can correct these defects. The increased levels of pA-site-stalled RNAPII in rat1-1 cells (8.2/8) (Fig. 2E; Supplemental S2C, middle panel) are not decreased by FCP1 overexpression or in the rpb1-N488D mutant. Hence, unusually high levels of Ser2P appear to specifically impact a subset of RNAPII that either fails to stall or is capable of resuming transcription after stalling.

\section{Rat1p displaces Ctk1p}

Increased CTD phosphorylation in rat1-1 cells and its suppression by extracopy Fcplp could be explained by defective recruitment of Fcp 1p in the absence of wt Rat1p activity. To test this possibility, we conducted YLR454W ChIP using myc-tagged Fcplp, which yielded specific signals at amplicons within the gene (Supplemental Fig. S3A). Unexpectedly, the relative ChIP efficiency of Fcp1-myc at the YLR454 and PMA1 genes was not decreased but was rather comparable to that of Rpb1p in both wt and rat1-1 cells at all measured positions (Fig. 3A; Supplemental Fig. S3B). Thus, Fcplp recruitment per transcription event is not affected by the rat1-1 mutation. While it is possible that the catalytic capacity of Fcp1p may be compromised in the rat11 background, we deemed it more likely that increased recruitment of a relevant kinase may explain the observed phenotype. We therefore chose to monitor levels of the main Ser2P kinase, Ctk1p, using 

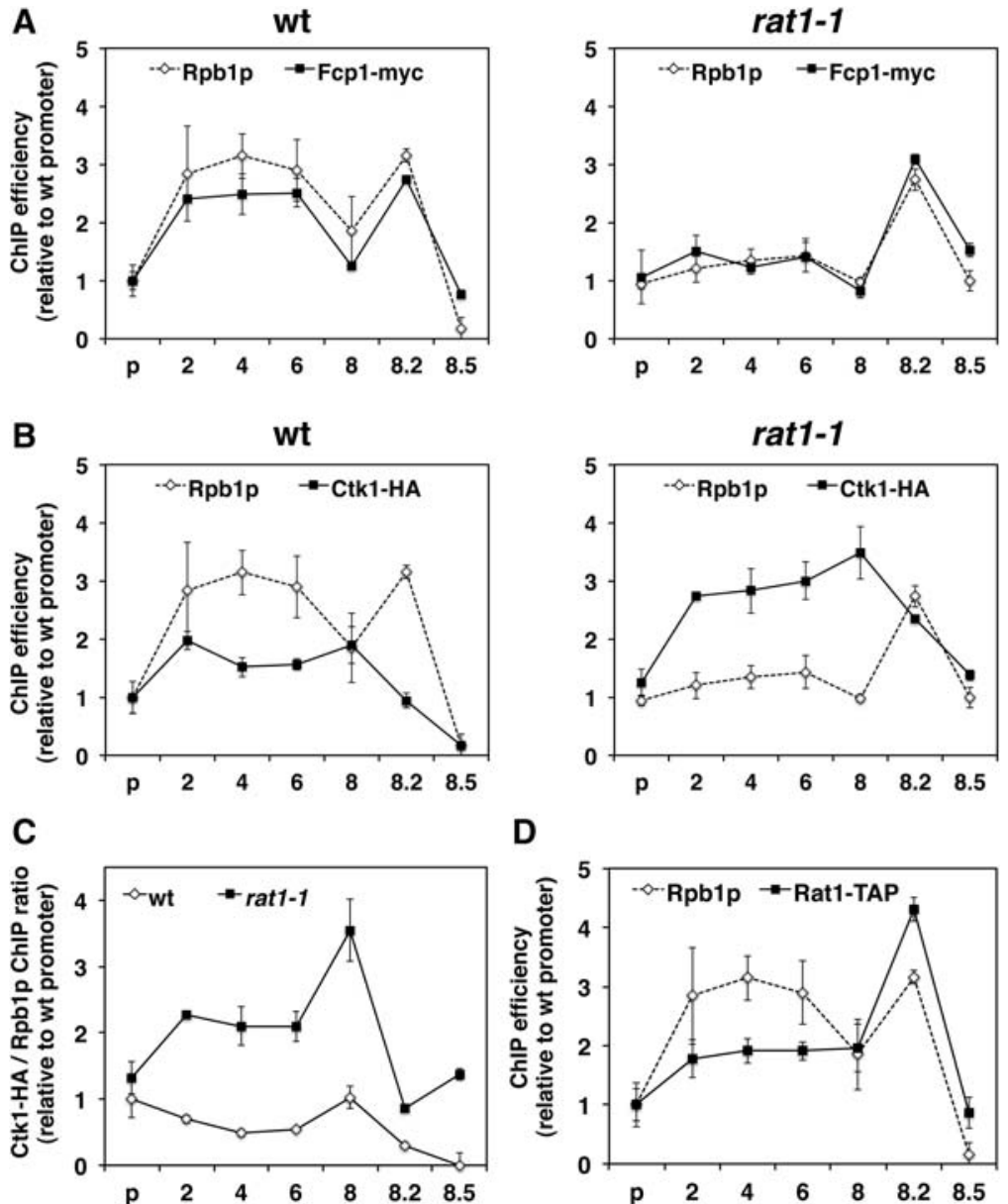

FIGURE 3. Ctk1-HA recruitment at GAL1::YLR454W is increased in rat1-1 cells. Fcp1-myc $(A)$, Ctk1-HA $(B)$, and Rat1-TAP $(D)$ distributions along the GAL1::YLR454W gene as measured by ChIP (solid lines). Parallel Rpblp ChIP values in the indicated strains are shown as dotted lines. Cells were grown as described in the legend of Figure 2B. ChIP values are relative to the wt signal at the YLR454W promoter region (P). (C) Ctk1-HA ChIP normalized to Rpblp in wt and rat1-1 strains. Position 8.5 in the wt strain is set to zero since Rpb1 ChIP levels are at background in wt cells (see Fig. 2B). Averages and standard deviations of all ChIP data were calculated from three independent biological experiments, each subjected to triplicate quantitative PCR analysis.

HA-tagged Ctk1p. Indeed, elevated Ctk1-HA occupancy per RNAPII could be observed in rat1-1 relative to wt cells at the YLR454W and PMA1 genes (Fig. 3B,C; Supplemental Fig. S3B). Hence, high Ser2P levels at chromatin in rat1-1 cells are likely due to increased recruitment of Ctk1p. This suggests a possible competition between cotranscriptional recruitment of Ratlp and Ctk1p. Indeed, Ctk1p becomes excluded from chromatin when RNAPII reaches the pA site where Ratlp levels peak (Fig. 3, cf. 8.2 amplicon in B, left panel, and D; Kim et al. 2004; Luo et al. 2006; Mayer et al. 2010). Notably, Ctk1p disappearance in that region is less efficient in rat1-1 cells; at the 8.2 position, a threefold increase of Ctk1p per RNAPII can be detected compared with the wt strain (Fig. 3C). The molecular basis for the competition between Ratlp and Ctk1p is unclear, but we speculate that they may compete for a common binding site, possibly on the CTD.

\section{RNAPII elongation rate is increased in the rat1-1 mutant}

Ratlp partakes in pre-mRNA $5^{\prime}$ capping quality control and causes premature transcription termination of RNAPII engaged with uncapped RNA (Jiao et al. 2010; Jimeno-Gonzalez et al. 2010). Under such conditions, the rat11 mutation leads to increased RNAPII levels inside gene bodies. Hence, the decreased RNAPII occupancy within gene bodies of rat1-1 cells, observed here without any impaired capping, is not explained by this role of Ratlp. On the other hand, Gu et al. (2013) recently found that substitution of CTD-Ser2 residues with alanines slows down transcription in mammalian cells. Thus, we speculated that CTD hyperphosphorylation in rat1-1 cells might cause an increased transcription elongation rate. In fact, this could help explain the lowered levels of gene body RNAPII in rat1-1 cells; i.e. the same amount of RNAPII is loaded at the promoter but spends less time transcribing along the coding region. To investigate this possibility, we monitored transcription kinetics of the GAL1-YLR454W system, where transcription rate can be extrapolated from the time it takes RNAPII to leave the gene template after turning off transcription by the addition of glucose to the culture (Mason and Struhl 2005). Hence, such a time course of the last wave of transcription was conducted by Rpblp ChIP analysis at different positions along the gene. Indeed, RNAPII took a shorter time to leave the YLR454W gene in the rat1-1 compared with the wt context (Fig. 4A). Therefore, RNAPII speed is higher in rat1-1 cells.

If an altered RNAPII elongation rate in the rat1-1 strain contributes to its growth defect, one might expect genetic interactions with other mutants affecting RNAPII speed. Indeed, combining the rat1-1 allele with the rpb1-E1103G mutation, which causes a faster transcription rate in vivo and in vitro (Malagon et al. 2006; Hazelbaker et al. 2013), resulted in an enhanced growth defect (Fig. 4B). Conversely, the rpb1-N488D or $r p b 2-10$ alleles, which both cause a slower RNAPII elongation rate in vivo and in vitro (Powell and Reines 1996; Mason and Struhl 2005; Jimeno-Gonzalez et al. 2010), suppressed rat1-1 thermosensitivity (Fig. 4C; Jimeno-Gonzalez et al. 2010). As a slowdown of RNAPII transcription downstream from the pA site is believed to 
A
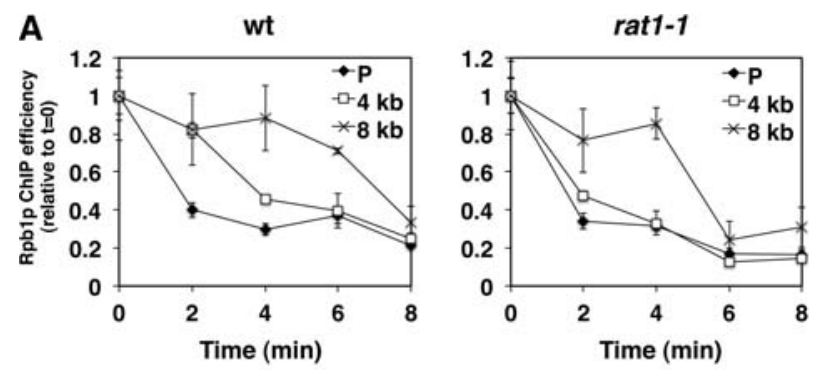

B
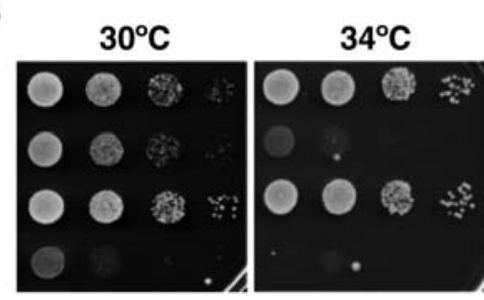

wt

rat1-1

rpb1-E1103G

rat1-1/rpb1-E1103G

C

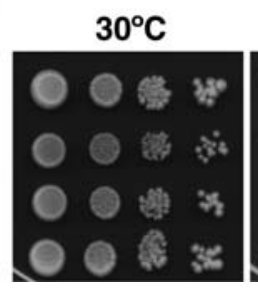

$34^{\circ} \mathrm{C}$

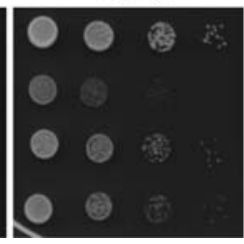

wt

rat1-1

rpb2-10

rat1-1/rpb2-10

FIGURE 4. Increased RNAPII transcription rate in rat1-1 cells. $(A)$ Transcription elongation rate assay measuring the last wave of transcription. Cells were grown in AA-Ura/ $2 \%$ raffinose at $30^{\circ} \mathrm{C}$ to an $\mathrm{OD}_{600}$ of 0.6. The GAL1 promoter was then induced with $2 \%$ galactose for $3 \mathrm{~h}$ at $30^{\circ} \mathrm{C}$. After an additional incubation for $1 \mathrm{~h}$ at $34^{\circ} \mathrm{C}$, the promoter was turned off by the addition of $2 \%$ glucose to the cultures, and samples were cross-linked at the indicated time points. Values correspond to Rpblp ChIP signals, obtained with the indicated amplicons, at each time point, and normalized to signals in galactose (time point 0 ). Averages and standard deviations of all ChIP data were calculated from three independent biological experiments, each subjected to triplicate quantitative PCR analysis. $(B, C)$ Growth capabilities measured by 10 -fold dilution series of the indicated strains spotted onto YM-1 plates. Strains were grown for $2 \mathrm{~d}$ at $30^{\circ} \mathrm{C}$ or $34^{\circ} \mathrm{C}$ as indicated. be a key step during the transcription termination process, an increased elongation rate may contribute to the termination defect observed in rat1-1 cells. Consistently, rescued growth of rat1-1 cells by introducing $r p b 1$ alleles slowing RNAPII progress is paralleled by a correction of the rat11 transcription termination phenotype (Jimeno-Gonzalez et al. 2010).

Ratlp is widely accepted to be involved in transcription termination. In addition, the enzyme is required for quality control of RNA $5^{\prime}$-end capping (Jiao et al. 2010; JimenoGonzalez et al. 2010). Previous reports all suggest a causal role for exonucleolytic attack of nascent RNA $5^{\prime}$ ends by Ratlp. Consistently, coexpression of a Rat1p catalytic dead mutant, in the rat1-1 strain, did not rescue CTD hyperphosphorylation (Supplemental Fig. S4), and exonucleolysis thus appears to also be required for the molecular phenotypes described here. As CTD phosphorylation impacts all steps of the transcription cycle, we suggest that the RNAPII CTD hyperphosphorylation phenotype of rat1-1 cells is taken into account when molecular phenotypes upon Ratlp inactivation are considered. How altered CTD phosphorylation status impinges on the various roles of Ratlp in transcription will be the focus of further investigation.

\section{MATERIALS AND METHODS}

\section{Yeast strains and plasmids}

Yeast manipulations and growth conditions were as previously described (Malagon et al. 2006). All strains used in this study are derived from a S288C background and listed in Table 1. The GAL1:: YLR454W reporter was introduced by crossing with the strain BY4741 GALlp::YLR454W (Mason and Struhl 2005). To isolate suppressors of rat1-1, we used the severe thermosensitivity phenotype conferred by the rat1-1/rpb1-E1103G double mutant at $34^{\circ} \mathrm{C}$. The strain Y2823 was transformed with a genomic library (a kind gift of François Lacroute) cloned into the URA3 containing

TABLE 1. Yeast strains

\begin{tabular}{|c|c|c|c|}
\hline Name & Strain & Genotype & Source \\
\hline rat1-1 rpb1-E1103G & Y2823 & MATa rat1-1 rpb1-E1103G & This study \\
\hline wt & Y2400 & MATa pGAL1-YLR454::URA3 ura3A & Jimeno-Gonzalez et al. (2010) \\
\hline rat1-1 & Y2402 & MATa rat1-1 pGAL1-YLR454::URA3 ura3A & Jimeno-Gonzalez et al. (2010) \\
\hline$r p b 1-N 488 D$ & Y2401 & MATa rpb1-N488D pGAL1-YLR454::URA3 ura3A & Jimeno-Gonzalez et al. (2010) \\
\hline rat1-1 rpb1-N488D & Y2403 & MATa rat1-1 rpb1-N488D pGAL1-YLR454::URA3 ura3A & Jimeno-Gonzalez et al. (2010) \\
\hline rpb1-E1103G & Y2820 & MATa rpb1-E1103G pGAL1-YLR454::URA3 ura3A & This study \\
\hline rat1-1 rpb1-E1103G & Y2823 & MATa rat1-1 rpb1-E1103G pGAL1-YLR454::URA3 ura3A & This study \\
\hline rpb2-10 & Y2571 & MATa rpb2-10 pGAL1-YLR454::URA3 ura3A & This study \\
\hline rpb2-10 rat $1-1$ & Y2572 & MATa rat1-1 rpb2-10 pGAL1-YLR454::URA3 ura3A & This study \\
\hline FCP1-myc & Y2517 & MAT $\alpha F C P 1-(m y c) 13:: K A N$ pGAL1-YLR454::URA3 ura3A & This study \\
\hline rat1-1 FCP1-myc & Y2518 & MATa rat1-1 FCP1-(myc) 13::KAN pGAL1-YLR454::URA3 ura3A & This study \\
\hline CTK1-HA & Y2515 & MATa CTK1-(HA)3::TRP1 pGAL1-YLR454::URA3 ura3A & This study \\
\hline rat1-1 CTK1-HA & Y2516 & MATarat1-1 CTK1-(HA)3::TRP1 pGAL1-YLR454::URA3 ura3A & This study \\
\hline RAT1-TAP & Y2411 & MATa RAT1-TAP::HIS3 pGAL1-YLR454::URA3 ura3A & Jimeno-Gonzalez et al. (2010) \\
\hline
\end{tabular}

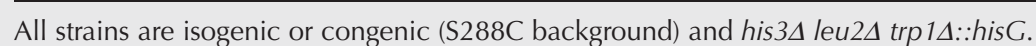


multicopy plasmid pFL44L (Bonneaud et al. 1991). Out of approximately 60,000 transformants, 14 clones suppressed the lack of growth at $34^{\circ} \mathrm{C}$ after reisolation of the original clones and retransformation. Sequencing of the clones identified 13 identical insertions containing the RAT1 gene (cXV coordinates 717670-721951; plasmid pRES21), while one clone carried a genomic fragment (cXIII 818791-822802) containing the FCP1 gene (plamid pRES7). Suppression was also observed in the rat1-1 single mutant, and no effect of RAT1 or FCP1 overexpression was observed for the rpb1E1103G single mutant. Hence, FCP1 is a high-copy suppressor of rat1-1. All results shown here were obtained using the FCP1 gene subcloned into the multicopy LEU2 containing vector pRS425. The plasmid expressing the rat1 exonuclease mutant pRS315 rat1D235A was kindly supplied by Steve Buratowski.

\section{ChIP analysis}

ChIP reactions were performed as previously described (JimenoGonzalez et al. 2006) by cross-linking with 1\% formaldehyde for $20 \mathrm{~min}$ at room temperature. IPs were carried out using the following reagents: Rpb1p, Sepharose-protein A beads (GE Healthcare) and the rabbit polyclonal antibody Rpb1-y80 (Santa Cruz Biotechnology); Rpb3, Dynabeads M-280 sheep anti-Mouse IgG (Invitrogen) and the mouse monoclonal antibody anti-POLR2C (Abcam); Rat1-TAP, Sepharose-IgG beads (GE Healthcare); Rpb1p CTD-Ser2P, Dynabeads M-280 sheep anti-Mouse IgM (Invitrogen) and the mouse monoclonal antibody H5 (Covance); Fcp1-myc, Dynabeads M-280 sheep anti-Mouse IgG (Invitrogen) and the mouse monoclonal antibody c-myc (Santa Cruz); and Ctk1-HA, Dynabeads M-280 sheep anti-Mouse IgG (Invitrogen) and the mouse monoclonal antibody anti-HA (Santa Cruz). Purified DNA was analyzed as previously described (JimenoGonzalez et al. 2010). Oligonucleotide sequences used for qPCR analysis of the GAL-YLR $454 W$ reporter gene are described by Jimeno-Gonzalez et al. (2010) and for PMA1 are as follows: 5, TCAGCTCATCAGCCAACTCAAG and CGTCGACACCGTGATT AGATTG; middle, TTGCCAGCTGTCGTTACCAC and TCGACA CCAGCCAAGGATTC; 3' UTR, TCTCTGGATGGTACTTTTTC TTTCTTG and TGCGTGTTGTGAATTGTGCTC; and T1, GC GCCCATACAGACA and CTTGTAGAATGGCCT.

\section{Antibodies for Western blotting}

The following antibodies were used for Western blotting analysis: mouse monoclonal antibody H14 (Covance); rat monoclonal antibodies 3E8, 4E12, and 3E10 (a gift from Dirk Eick); and rabbit polyclonal anti-Fcplp (a gift from Steve Buratowski). The other antibodies are as described for ChIP.

\section{SUPPLEMENTAL MATERIAL}

Supplemental material is available for this article.

\section{ACKNOWLEDGMENTS}

We thank Steve Buratowski and Dirk Eick for antibodies and plasmids. This work was supported by the Danish National Research Foundation (grant DNRF58) and the Novo Nordisk Foundation
(T.H.J.). M.S. is the recipient of a grant from the Lundbeck foundation.

Received July 4, 2013; accepted December 23, 2013.

\section{REFERENCES}

Ahn SH, Kim M, Buratowski S. 2004. Phosphorylation of serine 2 within the RNA polymerase II C-terminal domain couples transcription and $3^{\prime}$ end processing. Mol Cell 13: 67-76.

Archambault J, Chambers RS, Kobor MS, Ho Y, Cartier M, Bolotin D, Andrews B, Kane CM, Greenblatt J. 1997. An essential component of a C-terminal domain phosphatase that interacts with transcription factor IIF in Saccharomyces cerevisiae. Proc Natl Acad Sci 94: 14300-14305.

Bataille AR, Jeronimo C, Jacques PE, Laramee L, Fortin ME, Forest A, Bergeron M, Hanes SD, Robert F. 2012. A universal RNA polymerase II CTD cycle is orchestrated by complex interplays between kinase, phosphatase, and isomerase enzymes along genes. Mol Cell 45: 158-170.

Bonneaud N, Ozier-Kalogeropoulos O, Li GY, Labouesse M, MinvielleSebastia L, Lacroute F. 1991. A family of low and high copy replicative, integrative and single-stranded S. cerevisiae/E. coli shuttle vectors. Yeast 7: 609-615.

Calvo O, Manley JL. 2005. The transcriptional coactivator PC4/Sub1 has multiple functions in RNA polymerase II transcription. EMBO J 24: 1009-1020.

Chapman RD, Heidemann M, Albert TK, Mailhammer R, Flatley A, Meisterernst M, Kremmer E, Eick D. 2007. Transcribing RNA polymerase II is phosphorylated at CTD residue serine-7. Science 318: $1780-1782$.

Cho EJ, Kobor MS, Kim M, Greenblatt J, Buratowski S. 2001. Opposing effects of Ctk1 kinase and Fcp 1 phosphatase at Ser 2 of the RNA polymerase II C-terminal domain. Genes Dev 15: 3319-3329.

Danko CG, Hah N, Luo X, Martins AL, Core L, Lis JT, Siepel A, Kraus WL. 2013. Signaling pathways differentially affect RNA polymerase II initiation, pausing, and elongation rate in cells. Mol Cell 50: $212-222$.

Garcia A, Rosonina E, Manley JL, Calvo O. 2010. Sub1 globally regulates RNA polymerase II C-terminal domain phosphorylation. Mol Cell Biol 30: 5180-5193.

Ghosh A, Shuman S, Lima CD. 2008. The structure of Fcp1, an essential RNA polymerase II CTD phosphatase. Mol Cell 32: 478-490.

Gu B, Eick D, Bensaude O. 2013. CTD serine-2 plays a critical role in splicing and termination factor recruitment to RNA polymerase II in vivo. Nucleic Acids Res 41: 1591-1603.

Hazelbaker DZ, Marquardt S, Wlotzka W, Buratowski S. 2013. Kinetic competition between RNA polymerase II and Sen1-dependent transcription termination. Mol Cell 49: 55-66.

Jiao X, Xiang S, Oh C, Martin CE, Tong L, Kiledjian M. 2010. Identification of a quality-control mechanism for mRNA $5^{\prime}$-end capping. Nature 467: 608-611.

Jimeno-Gonzalez S, Gomez-Herreros F, Alepuz PM, Chavez S. 2006. A gene-specific requirement for FACT during transcription is related to the chromatin organization of the transcribed region. Mol Cell Biol 26: 8710-8721.

Jimeno-Gonzalez S, Haaning LL, Malagon F, Jensen TH. 2010. The yeast $5^{\prime}-3^{\prime}$ exonuclease Ratlp functions during transcription elongation by RNA polymerase II. Mol Cell 37: 580-587.

Kawauchi J, Mischo H, Braglia P, Rondon A, Proudfoot NJ. 2008. Budding yeast RNA polymerases I and II employ parallel mechanisms of transcriptional termination. Genes Dev 22: 1082-1092.

Kim M, Krogan NJ, Vasiljeva L, Rando OJ, Nedea E, Greenblatt JF, Buratowski S. 2004. The yeast Rat1 exonuclease promotes transcription termination by RNA polymerase II. Nature 432: 517-522.

Kim H, Erickson B, Luo W, Seward D, Graber JH, Pollock DD, Megee PC, Bentley DL. 2010. Gene-specific RNA polymerase II 
phosphorylation and the CTD code. Nat Struct Mol Biol 17: 12791286 .

Kobor MS, Archambault J, Lester W, Holstege FC, Gileadi O, Jansma DB, Jennings EG, Kouyoumdjian F, Davidson AR, Young RA, et al. 1999. An unusual eukaryotic protein phosphatase required for transcription by RNA polymerase II and CTD dephosphorylation in S. cerevisiae. Mol Cell 4: 55-62.

Komarnitsky P, Cho EJ, Buratowski S. 2000. Different phosphorylated forms of RNA polymerase II and associated mRNA processing factors during transcription. Genes Dev 14: 2452-2460.

Kuehner JN, Pearson EL, Moore C. 2011. Unravelling the means to an end: RNA polymerase II transcription termination. Nat Rev Mol Cell Biol 12: 283-294.

Lunde BM, Horner M, Meinhart A. 2011. Structural insights into cis element recognition of non-polyadenylated RNAs by the Nab3-RRM. Nucleic Acids Res 39: 337-346.

Luo W, Johnson AW, Bentley DL. 2006. The role of Rat1 in coupling mRNA 3'-end processing to transcription termination: Implications for a unified allosteric-torpedo model. Genes Dev 20: 954-965.

Malagon F, Kireeva ML, Shafer BK, Lubkowska L, Kashlev M, Strathern JN. 2006. Mutations in the Saccharomyces cerevisiae RPB1 gene conferring hypersensitivity to 6-azauracil. Genetics 172: 22012209.

Mandal SS, Cho H, Kim S, Cabane K, Reinberg D. 2002. FCP1, a phosphatase specific for the heptapeptide repeat of the largest subunit of
RNA polymerase II, stimulates transcription elongation. Mol Cell Biol 22: 7543-7552.

Mason PB, Struhl K. 2005. Distinction and relationship between elongation rate and processivity of RNA polymerase II in vivo. Mol Cell 17: 831-840.

Mayer A, Lidschreiber M, Siebert M, Leike K, Soding J, Cramer P. 2010. Uniform transitions of the general RNA polymerase II transcription complex. Nat Struct Mol Biol 17: 1272-1278.

Ozsolak F, Kapranov P, Foissac S, Kim SW, Fishilevich E, Monaghan AP, John B, Milos PM. 2010. Comprehensive polyadenylation site maps in yeast and human reveal pervasive alternative polyadenylation. Cell 143: $1018-1029$.

Powell W, Reines D. 1996. Mutations in the second largest subunit of RNA polymerase II cause 6-azauracil sensitivity in yeast and increased transcriptional arrest in vitro. J Biol Chem 271: 6866-6873.

Rasmussen EB, Lis JT. 1993. In vivo transcriptional pausing and cap formation on three Drosophila heat shock genes. Proc Natl Acad Sci 90: 7923-7927.

Schroeder SC, Schwer B, Shuman S, Bentley D. 2000. Dynamic association of capping enzymes with transcribing RNA polymerase II. Genes Dev 14: 2435-2440.

West S, Gromak N, Proudfoot NJ. 2004. Human 5' $\rightarrow 3^{\prime}$ exonuclease Xrn2 promotes transcription termination at co-transcriptional cleavage sites. Nature 432: 522-525. 

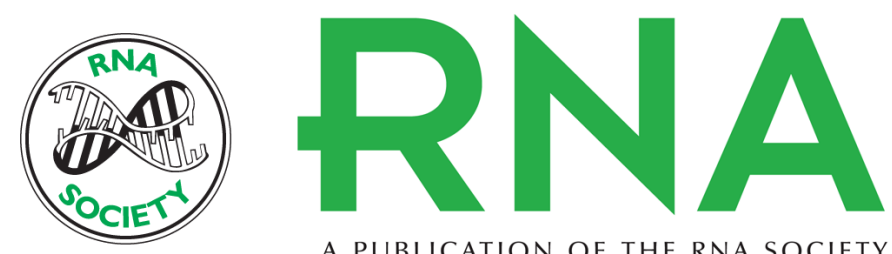

A PUBLICATION OF THE RNA SOCIETY

\section{Rat1p maintains RNA polymerase II CTD phosphorylation balance}

Silvia Jimeno-González, Manfred Schmid, Francisco Malagon, et al.

RNA 2014 20: 551-558 originally published online February 5, 2014

Access the most recent version at doi:10.1261/rna.041129.113

Supplemental Material

References

Creative Commons License

Email Alerting Service
http://rnajournal.cshlp.org/content/suppl/2014/02/04/rna.041129.113.DC1

This article cites 32 articles, 14 of which can be accessed free at: http://rnajournal.cshlp.org/content/20/4/551.full.html\#ref-list-1

This article is distributed exclusively by the RNA Society for the first 12 months after the full-issue publication date (see http://rnajournal.cshlp.org/site/misc/terms.xhtml). After 12 months, it is available under a Creative Commons License (Attribution-NonCommercial 3.0 Unported), as described at http://creativecommons.org/licenses/by-nc/3.0/.

Receive free email alerts when new articles cite this article - sign up in the box at the top right corner of the article or click here.

To subscribe to $R N A$ go to:

http://rnajournal.cshlp.org/subscriptions 